\title{
Quelques aspects de l'écriture de la violence dans le roman L'Attentat de Yasmina Khadra
}

\author{
Valentina RĂDULESCU \\ Université de Craïova \\ Roumanie
}

Résumé : La violence et ses effets dévastateurs interpellent plus que jamais les écrivains. Basé sur le roman L'Attentat (Julliard, 2005) de l'écrivain algérien Yasmina Khadra, cet article essaie de démontrer que l'écriture de la violence est, à la fois, la conséquence et le miroir des convulsions du monde contemporain. La démarche analytique est focalisée sur les fonctions de ce type d'écriture, sur les diverses formes de violence et leurs enjeux, ainsi que sur la mise en relation dans le roman d'un discours expliquant le terrorisme avec un discours humaniste, pacifiste.

\begin{abstract}
Violence and its devastating effects challenge writers more than ever. Based on the novel L'Attentat [The Attack] (Julliard, 2005), by the Algerian writer Yasmina Khadra, this paper aims to demonstrate that the writing of violence is both the consequence and the mirror of today's world convulsions. The analytical approach is focused on the functions of this type of writing, the various forms of violence and their problematic aspects, as well as on the relation of a discourse explaining terrorism with a humanist pacifist discourse, as it appears in the novel.
\end{abstract}

Mots-clés : écriture, violence, horreur, terrorisme, humanisme.

Keywords : writing, violence, horror, terrorism, humanism.

Le contexte socio-politique extrêmement tendu et mouvementé du monde contemporain, les crises émergentes ou qui perdurent «en ces temps de misères omniprésentes, de violences aveugles » (Cheng 2008, 13) ne cessent d’interpeller les écrivains. La violence, «le huitième péché capital» (Harrison 2015) devient tout naturellement une constante de l'écriture romanesque, le dénominateur commun de nombreux récits, qui se constituent dans de véritables "graphies de l'horreur»", dénonçant les atrocités cautionnées par une certaine idéologie, par l'appartenance religieuse, par la morale individuelle ou collective - autant de masques (parfois) de la barbarie humaine et de prétextes pour ses manifestations.

Très ancrés dans l'actualité, les romans de Yasmina Khadra (pseudonyme de Mohammed Moulessehoul) n'y font pas exception : Les Agneaux du Seigneur (1998) et À quoi rêvent les loups (1999) sont inspirés par « l'explosion de la violence qui dépasse l'entendement» (Bonn 1999, 5, cité par Daoud 2012, 96), due aux intégristes islamistes, qui a marqué l'Algérie au début de la dernière décennie du $\mathrm{XX}^{\mathrm{e}}$ siècle ; Ce que le jour doit à la nuit (2008)

\footnotetext{
${ }^{1}$ Le syntagme "graphies de l'horreur» a été créé par Rachid Mokhtari, dans son livre La graphie de l'horreur: essai sur la littérature algérienne, 1990-2000 (Alger, Éditions Chihab, 2002) et désigne les œuvres illustrant une écriture de l'urgence, née dans le contexte des violences intégristes qui ont bouleversé l'Algérie à partir de 1990.
} 
évoque des événements passés entre 1930 et 1962, dont la guerre de libération de l'Algérie; dans Les Hirondelles de Kaboul (2002), l'écrivain parle "du renoncement, de la cheptelisation de la nation, de la déshumanisation de la société talibane et surtout de l'Amour avec une majuscule » (Khadra 2007, S 18) ; dans L'Attentat (2005), il « explique la résistance palestinienne » (Ibidem), tandis que dans Les Sirènes de Bagdad (2006), il « raconte la guerre américaine vue par un jeune bédouin»(Ibidem). À la rentrée littéraire 2015, Yasmina Khadra continue de surprendre: dans La Dernière nuit du Raïs, il donne la parole au colonel Mouammar el Kadhafi pour raconter ses dernières heures avant l'exécution. L'œuvre de Yasmina Khadra comprend également des romans policiers, dont La Foire des Enfoirés (1993), Morituri (1997), Double Blanc (1997), L'Automne des chimères (1998) et des récits biographiques - L'Écrivain (2001), L'Imposture des mots (2002) - qui éclairent son trajet d'écrivain, sa conception de l'écriture, le rôle qu'il entend jouer dans le champ des lettres francophones algériennes (ou tout simplement de la littérature), l'écrivain étant bien connu pour son attachement profond à son algérianité, mais aussi à la langue française :

MCM : Vous n'allez pas de main molle pour exprimer votre amour de la langue française ("J'adore la langue française. Si elle était une femme, je l'aurais épousée ", affirmez-vous). Vous ne craignez pas que cet attachement viscéral au français ne vous conduise à revêtir l'habit du colonisateur?

$Y K$ : L'amour n'a rien à avoir avec l'identité. On peut être Algérien et épouser une Russe. Le cœur a ses raisons que la raison ignore. À aucun moment, le fait d'écrire en français n'a menacé mon algérianité. J'écris avec ma sensibilité d'Algérien, et rien d'autre. [...] La littérature est un texte, et non un territoire. (Khadra 2007, \16).

Même si dans la plupart de ses romans il dénonce la violence et ses conséquences dans diverses zones conflictuelles, Yasmina Khadra se défend d'écrire une simple littérature de témoignage ou de l'urgence, une telle approche de son œuvre étant réductrice, faussant sa véritable portée, occultant sa dimension esthétique ou l'originalité de son imaginaire :

Mes romans parlent de l'actualité, mais échappent aux témoignages. Ils restent des œuvres purement littéraires, avec un style singulier, une écriture reconnaissable, un rythme et une atmosphère particuliers. [...]

Contrairement à ce qu'avancent certains frères, qui pensent amoindrir l'importance de mon travail en le situant dans ce qu'ils appellent l'écriture de l'urgence (encore un caractère de complexés!), mes romans sont porteurs d'une âme, d'une émotion et d'un imaginaire exceptionnels. (Khadra 2007, \8).

Dans ce qui suit, notre réflexion portera, d'une part, sur les fonctions de la fiction, mises en relation avec l'écriture de la violence, qui se dégagent à la lecture du roman L'Attentat et, d'autre part, sur les diverses formes de violence présentes dans cette œuvre, afin de démontrer l'hypothèse que l'écriture de la violence est, à la fois, la conséquence et le miroir des convulsions du monde contemporain. 


\section{Présentation du roman}

Les hirondelles de Kaboul, Les sirènes de Bagdad et L'Attentat forment «la trilogie du malentendu des mal entendants » (Khadra 2007, \8), inspirée à son auteur par la nécessité de combattre «la perception complètement biaisée, pour ne pas dire stupide » (Khadra 2006, \1) du monde arabo-musulman et « d'œuvrer afin de reconstruire les passerelles naturelles qui ont toujours existé entre l'Orient et l'Occident» (Ibidem). Dans L'Attentat, l'action se déroule dans les villes de Tel-Aviv, de Bethléem, de Janin, autant d'espaces hautement symboliques du conflit israélo-palestinien et ce choix n'est certainement pas dû au hasard car

Le conflit entre Israël et Palestine est notre malheur commun. De même, la paix mondiale reste tributaire de ce qui se passe dans cette région. Les politiques doivent comprendre que tant que cette plaie est ouverte, la planète entière est menacée de gangrène. Ils doivent comprendre que la vie repose sur l'amour, le partage, sur la solidarité. (Khadra 2009, \12).

Narrateur et personnage central du récit, le médecin Amine Jaafari, Palestinien naturalisé Israélien, chirurgien réputé travaillant dans un hôpital de Tel-Aviv, est amené à soigner les victimes d'un attentat perpétré par une femme kamikaze dans un restaurant où une bonne partie des clients étaient des enfants participant à une fête. Ce n'est que le début de l'horreur : Amine apprend que le kamikaze n'est autre que sa femme, Sihem, et son geste inexplicable fait basculer sa vie. Au refus initial d'admettre que celle-ci, qu'il considère une simple victime, est l'auteur de l'acte terroriste, suit la certitude : une lettre de Sihem lui fait comprendre la vérité.

Dès lors, Amine doit affronter la cabale déclenchée contre lui à l'hôpital, les violences qu'il subit de la part des Israéliens, aussi bien que des Palestiniens parmi lesquels il enquête afin d'apprendre la vérité. Mais il doit surtout affronter ses propres tourments intérieurs, son incompréhension, son aveuglement et essayer de découvrir qui était vraiment celle qui avait partagé sa vie durant des années, les raisons de sa radicalisation et de son passage à l'acte.

Après l'enterrement de sa femme, meurtri jusque dans sa chair, blessé dans son orgueil d'homme amoureux ayant bâti tout son monde autour de la femme aimée et qui voit ce monde s'écrouler, Amine Jaafari commence une enquête acharnée : en seulement quelques jours, sur les traces de sa femme, il voyage de Tel Aviv à Bethléem, à Kafr Kanna, pour rencontrer ceux que Sihem avait croisés avant l'attentat et qui sont susceptibles de le renseigner. Il passe ensuite de l'autre côté du Mur, pour se rendre à Janin, la ville assiégée et c'est tout un autre monde en décomposition, subissant les pires atrocités, qu'il découvre, tout en renouant avec les siens, longtemps ignorés. Et c'est justement dans sa famille qu'il trouvera les réponses. Pourtant, même après la destruction par l'armée israélienne de la demeure de sa tribu (que songrandpère avait bâtie de ses propres mains) à la suite d'un nouvel attentat-suicide perpétré par son jeune neveu Wissam, même après la perte de tous ses repères 
familiers, Amine restera fidèle à son choix de médecin humaniste. La mort du personnage dans un attentat contre le Cheikh Marwan, le guide spirituel des intégristes, celui qui aurait béni Sihem à la veille de son acte, est symbolique : c'est l'affirmation de l'impossibilité de tout retour à la normalité, de lever le malentendu, le sacrifice inutile et absurde d'un innocent, l'échec (trop) prévisible du Bien devant le Mal.

\section{L'écriture de la violence et ses fonctions}

Écrire la violence n'est ni une fin en soi, ni un exercice de style gratuit. Cela exige une implication affective et intellectuelle particulière de celui qui s'attache à la décrire, fût-il historien ou auteur de fiction. Raphaëlle Branche note en ce sens : «accepter de travailler sur la violence, et prendre le risque de la décrire, nécessite d'accepter cette force dérangeante : elle a une puissance de bouleversement intime et dont il est vain d'espérer le contrôle total, chez soi comme, a fortiori, chez ses lecteurs » $(2010,29)$ et il est évident qu'on «ne peut pas faire l'économie du trouble que suscitent les récits de la violence» (Ibidem, 40), l'acte d'écrire, tout comme celui de lire, ce genre de fiction impliquant un état dysphorique.

Outre son côté déstabilisant, traumatisant, l'écriture de la violence remplit une série de fonctions qui recoupent celles de la fiction en général. Yasmina Khadra considère que « la fiction est la thérapie de la réalité » (2007, S 8) et il nous semble que cette fonction thérapentique est la plus importante dans les romans de la violence, qui assument « une sorte de prise en charge directe de la lourdeur du réel » (Charles Bonn 1999, 4, cité par Daoud 2012, 96). Mais de quelle réalité s'agit-il? Dans son analyse des trois romans du cycle du malentendu, Jędrzej Pawlicki constate que :

La stratégie narrative de Yasmina Khadra consiste en montrer les événements référentiels qui participent à la création d'une réalité propice au déclenchement de la violence. Les fictions khadraïennes sont contaminées par les événements réels dont le but est de mettre en relief les facteurs qui contribuent au basculement dans le sang. Les rares indices temporels dans la trilogie du grand malentendu fournissent un cadre général que l'écrivain remplit avec les événements fictifs pour créer une situation favorable au développement du terrorisme. $(2014,103)$.

Yasmina Khadra se sert de la métaphore du miroir pour décrire le rapport fiction-réalité ; pour lui, « la fiction a cette magie d'aller de l'autre côté du miroir et d'observer à son aise, et avec intelligence, ce qui se passe en face. Dans ce genre d'approche, elle ne pourrait se mettre à l'abri des éclaboussures. Elle devient un outil de réflexion et se met ainsi en danger. » (2007, \8). Il ne s'agit donc pas, chez l'écrivain algérien, de reproduire la réalité, mais de la confronter à elle-même à travers la fiction, de lui renvoyer une image critique. L'écriture de la violence confronte la réalité à ce qu'elle a d'abominable, exhibant l'impensable, racontant l'inimaginable. Il s'agit de dévoiler les avatars les plus monstrueux du Mal, avant et afin de pouvoir l'anéantir et en guérir. 
La fonction thérapeutique de l'écriture de la violence repose sur trois autres en étroite interdépendance: montrer la violence, y réfléchir et tenter de l'expliquer. Dans la vision de Yasmina Khadra,

La fiction permet d'esthétiser la banalité, de lui donner un sens et une portée, une dimension plus large, une audience plus crédible... La littérature est, par moments, un arrêt sur image. Elle donne à voir, à regarder de près, à aller au fond des choses, à pousser les portes dérobées des non-dits, de l'absurdité, de l'insoutenable. Paradoxalement, en nous initiant aux choses de la vie, elle nous apprend beaucoup sur nous-mêmes. Tous les personnages qu'elle nous propose nous renvoient à notre propre image, toutes les situations qu'elle nous raconte rejoignent notre propre existence. (2009, \$ 14).

L'Attentat contient de nombreuses scènes qui présentent en détail soit des actes de violence en train de se produire, soit les effets de ces actes, qui atteignent «l'horreur dans sa laideur absolue... $»^{2}$. Par exemple, au-delà du Mur, la ville de Janin offre l'image désolante d'une réalité modelée seulement par les mentalités détériorées des belligérants, menés par leurs "rancœurs assassines » $(A, 196)$ :

voir l'insoutenable de mes propres yeux me traumatise. À Tel-Aviv, j'étais sur une autre planète. Mes œillères me cachaient l'essentiel du drame qui ronge mon pays ; les honneurs que l'on me faisait occultaient la teneur véritable des horreurs en passe de transformer la terre bénie de Dieu en un inextricable dépotoir où les valeurs fondatrices de l'Humain croupissent, les tripes à l'air, où les encens sentent mauvais comme les promesses que l'on résilie, où le fantôme des prophètes se voile la face à chaque prière qui se perd dans le cliquetis des culasses et les cris de sommation. $(A, 196)$.

Montrer les dérives que la violence peut engendrer et leurs effets dévastateurs est également synonyme d'avertir contre la violence, contre le danger permanent qu'elle représente. À travers la voix d'Amine Jaafari, Yasmina Khadra met en garde contre « une Histoire scélérate, toujours prête à récidiver » $(A, 164)$.

Les fictions mettant en scène la violence sont également un espace de réflexion sur celle-ci et influencent la perception du phénomène chez le lecteur. L'Attentat a également une dimension explicative qui, malgré le fait que le roman est destiné à un double lectorat, semble l'orienter plutôt vers le lecteur occidental. Plusieurs sujets abordés dans le texte sont censés renseigner le lecteur peu familiarisé avec le monde musulman. Par endroits, le texte acquiert des accents didactiques et propose de véritables définitions de dictionnaire, telle la distinction islamiste - intégriste - combattant palestinien :

Un islamiste est un militant politique. Il n'a qu'une seule ambition : instaurer un État théocratique dans son pays et jouir pleinement de sa souveraineté et de son indépendance... Un intégriste est un djihadiste jusqu'au-boutiste. Il ne

2 Yasmina Khadra. L'Attentat. Paris : Pocket, 2011 [Julliard, 2005]. Les citations tirées du roman respectent la pagination de cette édition. Dans le corps de l'article, elles seront suivies désormais du titre abrévié du roman $(A)$ et du numéro de la page. 
croit pas à la souveraineté des États musulmans ni à leur autonomie. Pour lui, ce sont des États vassaux qui seront appelés à se dissoudre au profit d'un seul califat. [...] Nous ne sommes ni des islamistes ni des intégristes, docteur Jaafari. Nous ne sommes que les enfants d'un peuple spolié et bafoué qui se battent avec les moyens du bord pour recouvrer leur patrie et leur dignité, ni plus ni moins. $(A, 156)$.

Karl Ågerup parle d'une esthétique didactique chez l'écrivain algérien et considère que les romans de la trilogie du malentendu sont de véritables « didafictions », concept que le chercheur suédois crée pour désigner

un mode littéraire qui moyennant un jeu interdiscursif particulièrement conséquent fournit un enseignement à propos du monde historique ainsi que des perspectives qui le contemplent et qui le jugent. Cet enseignement, plutôt que de prendre une forme traditionnellement pédagogique, s'opère par des processus artistiques et multiformes qui intègrent l'encyclopédique et l'éthique dans le thématique et le personnel pour produire des effets esthétiques et didactiques spécifiques. $(2013,190)$.

S'inscrivant toujours dans cette logique, l'enquête d'Amine est également celle du lecteur : à partir du geste inexplicable de Sihem il s'agit de comprendre les mécanismes et les raisons qui transforment un individu en terroriste, de retracer «le cheminement d'une pulsation qui va se transformer en un geste abominable » (Khadra 2009, \ 7 ) :

J'ai voulu que tu comprennes pourquoi nous avons pris les armes, docteur Jaafari, pourquoi des gosses se jettent sur des cars comme sur des bonbonnières, pourquoi les cimetières sont saturés, pourquoi je veux mourir les armes à la main, pourquoi ton épouse est allée se faire exploser dans un restaurant. Il n'est pire cataclysme que l'humiliation. C'est un malheur incommensurable, docteur. Ça vous ôte le goût de vivre. Et tant que vous tardez à rendre l'âme, vous n'avez qu'une idée en tête: comment finir dignement quand vous avez vécu misérable, avengle et nu? $(A, 212)$.

Dès lors, la mort semble l'ultime salut pour les orphelins de leur pays, dont tous les rêves ont été éconduits. Si Yasmina Khadra aborde la question $\mathrm{du}$ terrorisme ce n'est pas pour le cautionner, mais pour essayer d'en surprendre les véritables ressorts et de combattre les stéréotypes du terrorisme comme seconde nature de l'Arabe ou du musulman ou celui du terroriste envisagé comme un cas pathologique. Le romancier insiste aussi sur le détachement total du réel - une sorte de transe irréversible, à laquelle accède le terroriste avant son geste de folie assumée et qui marque aussi le moment de rupture, l'entrée dans une autre réalité, dans une zone de non-retour :

Je crois que même les terroristes les plus chevronnés ignorent vraiment ce qu'il leur arrive. Un déclic dans le subconscient et c'est parti. [...] Tu crois n'en faire qu'à ta tête, mais ce n'est pas vrai. T'es rien d'autre que l'instrument de tes propres frustrations. Pour toi, la vie, la mort, c'est du pareil au même. Quelque part, tu auras définitivement renoncé à tout ce qui pourrait donner une chance à ton retour sur terre. Tu planes. Tu es un extraterrestre. Tu vis dans les 
limbes, à traquer les houris et les licornes. Le monde d'ici, tu ne veux plus en entendre parler. Tu attends juste le moment de franchir le pas. $(A, 95)$.

Plusieurs personnages tiennent le long du roman des propos qui semblent légitimer le recours à la violence et justifient les actions au nom de la cause palestinienne. À ces propos s'oppose le discours humaniste du docteur Jaafari, qui souligne à certains moments la nécessité de sauver, de préserver et de célébrer la vie :

Je ne me reconnais pas dans ce qui tue, ma vocation se situe du côté de ce qui sauve. $(A, 224)$

Pour moi, la seule vérité qui compte est celle qui m’aidera un jour à me reprendre en main et à retrouver mes patients. Car l'unique combat en quoi je crois et qui mériterait vraiment que l'on saigne pour lui est celui du chirurgien que je suis et qui consiste à réinventer la vie là où la mort a choisi d'opérer. ( $A$, 226).

Yasmina Khadra illustre donc dans son roman des positions politiques et idéologiques irréconciliables, il essaye de remonter aux sources du malentendu entre Israéliens et Palestiniens. Tout en procédant de la sorte, le romancier garde sa neutralité, se dissimule derrière ses personnages, les laisse porter seuls leurs histoires, leurs messages, mais ne se situe jamais ouvertement sur une position partisane d'une attitude politique ou d'une autre. L'écrivain réitère constamment sa distanciation, le désir que le lecteur reste le seul juge de ses œuvres, mais prend soin de lui fournir «un maximum d'ingrédients pour qu'il se fasse sa propre idée et je le laisse libre de décider» (Khadra, 2007, S 8). Cette distanciation est aussi celle du narrateur, dont Yasmina Khadra fait à plusieurs reprises son porte-parole: "Je ne me souviens pas d'avoir applaudi le combat des uns ou condamné celui des autres, leur trouvant à tous une attitude déraisonnable et navrante. » $(A, 163)$.

\section{La violence et ses formes}

Deux conceptions de la violence s'actualisent dans le roman khadraïen : une «conception instrumentale de la violence » selon laquelle « la violence est le moyen à travers lequel atteindre des objectifs déterminés » (Corradi 2009, 1) et une conception que Consuelo Corradi nomme moderniste, selon laquelle «la violence est une force sociale chargée de signification et douée d'une capacité structurante de la réalité » (Ibidem), qui «modèle culturellement les corps des victimes et des agresseurs» (Ibidem, 3). Nous verrons ensuite sous quelles formes se manifeste la violence dans le roman L'Attentat.

L'attentat en tant qu'acte terroriste est, à notre avis, la manifestation extrême de la violence susceptible de créer le plus de dégâts matériels et de chocs émotionnels au sein d'une communauté locale ou de la communauté internationale. Susceptible aussi de les déstabiliser profondément, d'induire et de maintenir un sentiment d'insécurité, par la menace permanente qu'il représente, par son imprévisibilité, rappelant constamment à l'humanité ses limites et ses frayeurs. 
Le roman khadraien débute par la description d'un double attentat, et l'écrivain recourt à une technique narrative qui consiste à

faire venir un affect à l'état presque pur et dont le premier effet est de traumatiser, c'est-à-dire de tétaniser les sens et la pensée. Il s'agit bien de mettre le lecteur en état de choc. Pour tenter de l'en tirer plus ou moins vite, ensuite, par des éléments explicatifs et une manière d'universalisation ou de relativisation... (Meitinger 2004, 1).

L'incipit in medias res présente le moment où une explosion se produit et le chaos s'installe. Le lecteur est confronté d'emblée à «la violence dans ses conséquences physiques brutales et irrémédiables, dans sa monstruosité immédiate » (Ibidem) et ses seules informations sont celles que le narrateur lui fournit en narration simultanée, comme dans un reportage en direct. Il s'agit d'un attentat contre le cheikh Marwan, dont Amine Jaafari est aussi victime. Les circonstances de l'attentat ne seront révélées que beaucoup plus tard, mais au début du roman, c'est sur la scène apocalyptique que la narration se focalise : des corps déchiquetés, des personnes brûlées vives, la voiture du cheikh en flammes, les hurlements des blessés qui interfèrent avec les sirènes des ambulances venues porter secours, tout crée un paysage cauchemardesque :

Une crue de poussière et de feu vient me happer, me catapultant à travers mille projectiles. J'ai le vague sentiment de m'effilocher, de me dissoudre dans le souffle de l'explosion.... À quelques mètres - ou bien à des années-lumière, le véhicule du cheikh flambe. Des tentacules voraces l'engloutissent, répandant dans l'air une épouvantable odeur de crémation. Leur bourdonnement doit être terrifiant ; je ne le perçois pas. Une surdité foudroyante m'a ravi aux bruits de la ville. Je n'entends rien, ne ressens rien. Je ne fais que planer, planer. Je mets une éternité à planer avant de tomber par terre, groggy, démaillé, mais curieusement lucide, les yeux plus grands que l'horreur qui vient de s'abattre sur la rue. $(A, 8)$.

Progressivement, de l'événement la narration se focalise sur l'agonie des victimes : "près de moi, un vieillard défiguré me fixe d'un air hébété : il ne semble pas se rendre compte que ses tripes sont à l'air, que son sang cascade vers la fondrière » $(A, 9)$, dont le narrateur : "Ma jambe repose contre mon flanc, grotesque et horrible à la fois ; un mince cordon de chair la retint encore à ma cuisse. D'un seul coup toutes mes forces me désertent. J'ai le sentiment que mes fibres se dissocient les unes des autres, se décomposent déjà...» $(A$, 10).

Même si des indices lui permettent de la pressentir, le lecteur n'a pas encore la certitude de la mort du narrateur. Yasmina Khadra opte pour un dévoilement retardé, qui lui permet, à la fois, d'accroître la tension narrative et de maintenir l'incertitude du lecteur, lui suggérant une possible chance de survie d'Amine : entassé dans une ambulance, parmi d'autres cadavres, celui-ci prie : «Dieu, si c'est un affreux cauchemar, faites que je me réveille et tout de suite...» $(A, 11)$. 
La prière d'Amine clôt la première partie du récit, qui passe brusquement à sa deuxième partie, en fait une longue analepse qui retracera l'enchaînement des faits et le trajet du personnage, jusqu'au moment de l'attentat qui ouvre le récit et qui le referme aussi, dans une narration circulaire. Après une brève séquence qui met en place le cadre du récit et une partie des personnages, la description du deuxième attentat accentue et fait perdurer le choc initial du lecteur. Les images de l'atrocité sont intensifiées par celles de la haine, révélant la véritable dimension des relations entre Israéliens et Palestiniens :

Un blessé exige que l'on s'occupe immédiatement de lui. Il a le dos écorché d'un bout à l'autre et une partie de l'omoplate à nu. [...]

D’un seul coup, le corps du blessé se raidit [...]. En une fraction de seconde, ses traits congestionnés se défont de leur douleur et cèdent la place à une expression démente, faite de rage froide et de dégoût. [...]

- Je ne veux pas qu'un Arabe me touche, grogne-t-il en me repoussant d'une main hargneuse. Plutôt crever. $(A, 20-21)$.

Dans l'ensemble du roman, cette stratégie narrative, qui fait alterner les moments de tension intense avec des scènes qui atténuent légèrement la tension, autant au niveau de la narration que de l'acte de lecture, est très porteuse car l'intérêt du lecteur est constamment maintenu et le récit ne perd rien en dynamisme et en intensité.

À la fin du roman, le fil des événements aboutit au moment où il avait été suspendu dans la scène initiale, et continue dans un mélange inouï d'horreur et de poésie tragique : à la fois présent sur la scène de l'attentat et détaché du monde des vivants, veillé par l'image de la mère qui émerge d'un rideau de fumée, Amine se contemple avant et après la mort, enregistre et narre ses sensations, le moment du passage et son expérience de la mort :

Seul un vieillard s'agenouille devant moi. Il évoque le nom du Seigneur, porte la main sur mon visage, baisse mes paupières. D'un coup toutes les lumières et tous les bruits du monde s'estompent. Une peur absolue me saisit. Pourquoi me ferme-t-il les yeux ?... C'est en n'arrivant pas à les rouvrir que je comprends : C'est donc ça; c'est fini, je ne suis plus.

Dans un ultime sursaut, je veux me reprendre en main ; pas une fibre ne frémit en moi. Il n'y a plus que cette rumeur cosmique qui bourdonne, m'investit cran par cran, me néantise déjà... $(A, 245)$.

Ce n'est pourtant pas sur l'image de la mort que le récit s'achève, mais sur celle du décollage vers l'éternité, sur le moment où Amine, retrouvant son âme et son innocence d'enfant, guidé par la voix de son père, tout en accédant à une autre dimension, traverse un monde où le bonheur serait encore possible, détruisant, symboliquement, ce qui l'entrave - le Mur. C'est dans une explosion de vitalité, de lumière et de rêve qu'Amine vainc l'opacité et les ténèbres :

Une aurore boréale se lève sur les vergers en fête; les branches se mettent aussitôt à bourgeonner, à fleurir, à ployer sous leurs fruits. L'enfant longe les 
herbes folles et fonce sur le Mur qui s'effondre telle une cloison en carton, élargissant l'horizon et exorcisant les champs qui s'étalent sur des plaines à perte de vue... ( $A, 245)$.

Les diverses formes de violence s'alimentent les unes les autres. Ainsi, à la suite de l'attentat de Sihem, Amine est marginalisé par les communautés auxquelles il s'était intégré et subit diverses agressions. Dans son hôpital, malgré le soutien d'une partie des patients ou d'amis de longue date, comme Kim Yehuda, il sera déclaré persona non grata. Sa maison est vandalisée, il est isolé par son voisinage, agressé physiquement par " une meute surexcitée » $(A$, 62), qui le traite de «Sale terroriste ! Fumier! Traître d'Arabe !» $(A, 62)$. Tous ces stéréotypes violemment réactivés, la concertation des diverses communautés dans l'exclusion sociale du personnage montrent en fait que leur ouverture à l'autre, qui est rejeté comme un corps étranger, l'acceptation de son intégration et la réconciliation ne sont qu'un leurre.

En outre, les Palestiniens marginalisent aussi Amine. Si, à son retour, il est bien accueilli par sa famille, les combattants palestiniens qu'il croise soulignent le clivage de leurs positions :

Nous sommes dans un monde qui s'entre-déchire tous les jours que Dieu fait. Notre patrie est violée à tort et à travers, nos enfants ne se souviennent plus de ce qu'école veut dire, nos filles ne rêvent plus depuis que leurs princes charmants leur préfèrent l'Intifada, nos villes croulent sous les engins chenillés et nos saints patrons ne savent plus où donner de la tête ; et toi, simplement parce que tu es bien au chaud dans ta cage dorée, tu refuses de voir notre enfer $(A, 158)$.

Il est clair, nous n'empruntons pas le même chemin. Nous passerions des mois et des années à essayer de nous entendre qu'aucun de nous ne voudrait écouter l'autre. $(A, 160)$.

Le personnage oscille donc entre deux univers qui lui sont aussi hostiles, où il ne trouve plus de points d'ancrage. Sans atteindre des formes excessives, la torture physique s'associe à l'exclusion sociale. Soupçonné d'une possible complicité avec sa femme, Amine Jaafari est soumis à un interrogatoire brutal, pendant lequel on lui applique des traitements dégradants :

Ma chemise trempée de sueur me ronge le dos avec la voracité d'un bouquet d'orties. J'ai faim, j'ai soif, j'ai mal et nulle part je ne vois le bout du tunnel. On a dû me porter par les aisselles pour m'emmener pisser. J'ai vidé la moitié de ma vessie avant que je parvienne à ouvrir la braguette. Pris de nausée, j'ai failli me casser la figure sur le bidet. On m'a carrément traîné en me ramenant dans ma cage. Ensuite, de nouveau le harcèlement, les questions, les coups de poing sur la table, les petites gifles pour m'empêcher de tourner de l'œil. $(A, 49)$.

Innocenté, le personnage ne se sent pas davantage en sécurité, il continue de se sentir traqué, de ressentir l'hostilité et la cruauté des officiers des services secrets israéliens, très bien rendue par une métaphore animale: «ils ont l'air d'une horde de loups regardant s'éloigner la proie qu'ils croyaient avoir piégée » $(A, 54)$. 
Le cauchemar continue en territoire palestinien, où il est incarcéré pendant une semaine dans des conditions inhumaines, afin de comprendre l'humiliation de son peuple, tout en subissant lui-même l'humiliation extrême. Tous ces traitements le déstabilisent, mais, même s'il arrive à éprouver des instants de pure haine, il ne renonce pas à ses principes humanistes. Pourtant, sa descente en enfer continue : dans sa quête obsessionnelle du signe qu'il n'a pas su comprendre de la part de Sihem et terrifié à l'idée de sa possible trahison, il n'arrive pas à faire son deuil, il n'est plus nulle part chez lui et, inexorablement, il est broyé par les mécanismes de la fatalité enclenchés au moment de l'attentat.

Une forme de violence particulière dans le roman est représentée par les représailles de l'armée israélienne contre la tribu d'Amine, après un nouvel attentat-suicide contre un poste de garde, perpétré par son neveu Wissam, fier de tomber sur le champ d'honneur. Une action brutale, rapide, efficace, qui en quelques minutes efface tous les repères ancestraux d'une famille. Amine, résigné, ne peut que contempler «le tas de décombres qui fut, sous un ciel étincelant, il y a des années-lumière, mon château de petit prince à pieds nus. [...] Il a suffi d'un bull pour réduire en poussière, en quelques minutes, l'éternité entière. » $(A, 239)$. Il s'agit là d'un nouvel exemple de violences qui s'engendrent réciproquement, dans un rythme infernal, angoissant, dans un perpetuum mobile de la cruauté, toujours plus gourmand.

Le roman rappelle aussi, discrètement, la Shoah: le vieux Yehuda, grand-père de Kim, est un rescapé des atrocités nazies. Il évoque fragmentairement le drame de sa famille, se contente de suggérer les épreuves subies. Le personnage est symbolique d'un autre point de vue: dans un univers convulsionné, il montre comment on peut gérer le traumatisme, comment on peut exorciser le mal et être en paix avec soi-même :

Il faut toujours regarder la mer. C'est un miroir qui ne sait pas nous mentir. C'est aussi comme ça que j'ai appris à regarder derrière moi. Avant, dès que je jetais un coup d'œil par-dessus mon épaule, je retrouvais intacts mes chagrins et mes revenants. Ils m'empêchaient de reprendre goût à la vie, tu comprends ? Ils gâchaient mes chances de renaître de mes cendres... [...] Qui regarde la mer, tourne le dos aux infortunes du monde. Quelque part, il se fait une raison. $(A, 81)$.

Dans cette superbe leçon de dignité et de force morale s'actualise, par une subtile mise en abîme, l'idée de la littérature comme " outil d'apaisement » (Khadra 2009, \14), essentiel pour contrecarrer la violence, pour réapprendre à vivre à ceux qui ont traversé l'enfer.

\section{Conclusions}

Si les fictions de la violence, tout en révélant des facettes des plus tragiques de l'histoire, sont peu susceptibles d'en influencer visiblement l'évolution à court terme, elles sont, en revanche, des instruments essentiels qui agissent sur les consciences et peuvent engendrer progressivement des prises de position concrètes et des changements significatifs à tous les niveaux 
de la société. Elles sont des espaces de mémoire remarquables où, comme il advient de plus en plus souvent dans les romans contemporains, la parole est donnée à tous, victimes ou bourreaux, multipliant ainsi les chances de comprendre les mécanismes intimes des manifestations de la violence et la véritable dimension de leur force destructive. Elles inscrivent des événements et des drames fictionnellement interprétés ou purement fictionnels dans la mémoire collective de l'humanité, leur conférant, au-delà de la coloration tragique et poétique, l'épaisseur du vécu, qui échappe souvent à l'historien.

\section{Références bibliographiques}

Ågerup, Karl. Didafictions. Littérarité, didacticité et interdiscursivité dans douze romans de Robert Bober, Michel Houellebecq et Yasmina Khadra. Stokholm : US-AB, 2013.

Branche, Raphaëlle. «La violence coloniale. Enjeux d'une description et choix d'écriture ». Tracés. Revue de Sciences humaines, 19 | 2010. [En ligne]. Mis en ligne le 30 novembre 2012. URL: http://traces.revues.org/4866. DOI : 10.4000/traces.4866. (Consulté le 29 juillet 2015).

Cheng, François. Cinq méditations sur la beauté. Nouvelle édition revue et corrigée. Paris : Albin Michel, 2008 [2006].

Corradi, Consuelo. "Violence, identité et pouvoir». Socio-logos. Revue de l'association française de sociologie. [En ligne]. Mis en ligne le 4 mai 2009. URL : http://sociologos.revues.org/2296. (Consulté le 3 août 2015).

Daoud, Mohammed. "La littérature et la communication interculturelle». Agapes Francophones. Études de lettres francophones réunies par Andreea Gheorghiu (responsable du volume et du Colloque 2012), Ramona Malița, Ioana Marcu, Mariana Pitar, Dana Ungureanu. Actes du Colloque "Passeurs de mots », des 16 et 17 mars 2012, organisé par la Chaire de français et le Centre d'Études Francophones de l'Université de l'Ouest de Timişoara. Timişoara : Éditions de l’Université de l'Ouest, 2012 : 87-97.

Harrison, Jim. Entretien (avec François Busnel) sur la sortie de son roman Péchés capitaux, diffusé dans le cadre de l'émission La Grande Librairie, sur France 5, le 3 septembre 2015. [En ligne]. Mis en ligne le 3.09.2015. URL: http://www.france5.fr/emissions/la-grande-librairie. (Consulté le 4.09.2015).

Khadra, Yasmina. "Aller au commencement du malentendu». Entretien (avec Christine Rousseau). Le Monde des Livres. [En ligne]. Mis en ligne le 28. 09. 2006. URL : sur http://www.lemonde.fr/livres/article/2006/09/28/yasminakhadra-aller-au-commencement-dumalentendu_817959_3260.html\#SqgiVz6X 3AsQkKkb.99. (Consulté le 12 août 2015).

Khadra, Yasmina. Entretien (avec Mohamed Chafil Mesbah). Le Soir d'Algérie: «Entretien du mois». [En ligne]. Mis en ligne le 26. 04. 2007. URL: http://www.lesoirdalgerie.com/articles/2007/04/26/article.php?sid=52831\&c id=30. (Consulté le 12 août 2015).

Khadra, Yasmina. «Le choc des cultures: un choc d'incultures » (propos recueillis par Didier Billion et Marie de Jerphanion le 11 mars 2009). Revue Internationale et Stratégique $\mathrm{n}^{\circ} 74$ (2/2009) : 7-13. [En ligne]. URL: www.cairn.info/revueinternationale-et-strategique-2009-2-page-7.htm. DOI : 10.3917/ris.074.0007. (Consulté le 12 août 2015). 
Meitinger, Serge. «Écriture de la violence, violence de l'écriture: Le cas des jeunes écrivains malgaches ». [En ligne]. Mis en ligne le 16 février 2004 sur le site CRITAOI de l'AUF. URL: http://critaoi.org/bib/archive/ 00085/02/PMO.001.pdf. (Consulté le 2 août 2015).

Mokhtari, Rachid. La graphie de l'horreur: essai sur la littérature algérienne, 1990-2000. Alger : Éditions Chihab, 2002.

Pawlicki, Jędrzej. «La trilogie du grand malentendu de Yasmina Khadra : implication plurielle des héros khadraïens ». Planeta Literatur. Journal of Global Literary Studies 1/2014. [En ligne]. URL: http://www.planeta-literatur.com/uploads /2/0/4/9/20493194/pl_1_2014_103_121.pdf. pp. 103-122. (Consulté le 7 août 2015).

\section{Corpus}

Khadra, Yasmina. L'Attentat. Paris : Pocket, 2011 [Julliard, 2005]. 
